\title{
Implementing Medical Business Processes Integrating Server Technologies
}

\author{
Ivan Zoraja ${ }^{1}$, Vojimir Golem² and Boris Ilijić ${ }^{1}$ \\ ${ }^{1}$ FESB, Department of Electronics and Computer Science, University of Split, Croatia \\ 2 MicroGolem, Split, Croatia
}

\begin{abstract}
In this paper we describe a BPM solution implemented by integrating server technologies in a SOA manner. Our solution empowers healthcare workers to more efficiently and effectively create clinical forms and contribute in clinical business processes. The clinical forms are XML documents created either using a special editor or web forms. Shared access and document management facilities are supported via the SharePoint Services while business processes management is driven by the BizTalk server. The integration between servers and components is realized via Web Services, adapters, and event handlers. The core logic behind business processes is implemented via BEPL constructs that obtain clinical forms, perform the requested data transformations, store data into the database, and push forms further into business processes. Event handlers receive forms from processes and, according to the routing information, deliver them to recipients. Web services provide SOA glue and lookups.
\end{abstract}

Keywords: BPM, SOA, HL7, EHR, BPEL, BizTalk, SharePoint

\section{Introduction}

Traditional paper work in healthcare companies and related business processes are usually complex and tedious to use since too much time was spent on entering, collecting, obtaining, verifying, exchanging, and reporting commonly used information. Clinical Forms' Manager (CFM) is a Business Process Management (BPM) solution that provides an easy management of clinical forms and corresponding business processes allowing various healthcare professionals in the healthcare industry to automate paper work using an elegant, yet efficient software solution.
CFM integrates server technologies to implement business processes and therefore reduces time needed to specify, design, construct, deploy, and manage such class of applications. From the developers' perspective, server technologies greatly reduce manual coding and therefore ensure shorter development cycles.

Our clinical forms are in XML format and can be created either using the InfoPath editor or ASP.NET pages. SharePoint Services are used to implement web-based shared accesses as well as basic document and workflow management facilities, while the BizTalk server is used to drive core business processes. XML Web Services provide the necessary glue to build Service-oriented Architecture (SOA) solutions. Currently, CFM is implemented using .NET technologies and enterprise servers, but since CFM is based on the Business Process Execution Language (BEPL), it can be ported to other platforms that support BEPL such as JBoss.

\subsection{Business Opportunity}

The CFM represents a technological part of the broader health management initiative aimed at optimizing and improving the usage of health services via Internet with centralized management, marketing, and distribution. This project opens opportunities to market interfaces to interested third parties such as insurance agencies and pharmacies. Via its advanced design, architecture, and implementation, CFM provides a platform for a set of healthcare services that 
are geared to create a well managed and orchestrated healthcare framework out of a large number of independent providers with disparate systems. CFM can be used to connect other medical information systems since it is based on core healthcare and technology standards.

\section{Software Requirements}

The CFM's software requirements are driven by the needs of a real healthcare organization that offers healthcare services and targets small and medium size healthcare providers like physician's offices and clinical laboratories on one end, and potential patients, including foreigners, without sufficient healthcare insurance, as well as demographic segment that can afford additional healthcare insurance.

\subsection{Actors and Features}

The main stakeholders affected by our solution can be roughly categorized as the ones who offer healthcare services and those who use the services - all of them communicate by exchanging electronic forms (medical documents). More specifically, CFM supports five types of actors that can contribute in clinical business processes and obtain business value: a patient is a person subscribed by a healthcare organization, a provider is a doctor specialist who can offer a healthcare service, ePharmacy represents a pharmacy that is able to exchange electronic medical documents, insurance represents an insurance company, and a broker is a doctor who arranges the interactions. The system can be configured with one or more brokers - usually a city is managed by a single broker that communicates the others to offer intercity services. The following figure represents the general model of CFM.

Participants in clinical business processes use CFM to easily create and fill out medical documents, as well as to automate and improve their business interactions by reducing the need to re-enter the same information each time it is needed. Information in CFM can be accessed, maintained, received, directed, and shared among different actors in XML format to simplify data exchange. CFM gives healthcare users sophisticated access to information so they

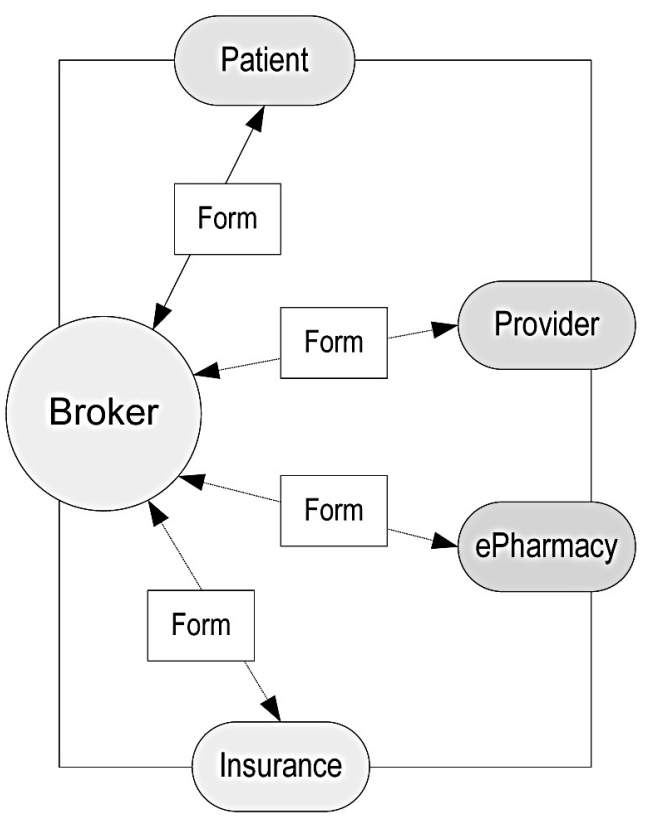

Figure 1. The general model od CFM.

can gain deeper insights into medical procedures and take more effective actions.

CFM supports rapid development of rich medical forms and building of custom solutions on flexible applications and servers and therefore makes it easy to perform necessary modifications when business requirements change. Healthcare organizations and employees using CFM can work together with speed and agility collaborating on multiple medical documents and improving individual productivity. Finally, CFM provides a central management of the deployment and security for all users and applies current industry standards and protocols like XML, WSDL, SOAP, the WS-* standards, and HL7 [12] to flexibly connect end users with business processes.

\subsection{Typical Business Process}

CFM supports the creation and exchange of clinical forms via corresponding BPEL-based [1] business processes. In a typical CFM business process, the broker examining the patient, makes an appointment with the appropriate specialist, creates a clinical form, and sends it to the doctor specialist (provider). The specialist examines the patient, creates a clinical form about his findings and results, and sends them back to the broker. All CFM forms are digitally signed 
and are stored in the archive of the particular patient who can later access them. Based on the results from the specialist, the broker, if needed, creates a prescription and sends it to a pharmacy. He then creates a report and sends it to the insurance company. If needed, the broker can create other clinical forms and start another round-trip in the context of the same patient episode.

\subsection{User Experience}

CFM allows healthcare workers to easily collect patient information into clinical forms, using a desktop PC or tablet PC. CFM can upload/download other required information to/ from document repositories where it is instantaneously available, greatly eliminating the need of searching the patient's paper-based assessment and reducing his/her wait time. In addition, using a single set of patient assessment forms eliminates duplicate treatment orders and information requests, and thereby reduces redundancy.

In Figure 2, we show the basic CFM use cases for a broker. $\mathrm{He} / \mathrm{She}$ can fill out a form using two types of editors. The former provides rich controls, integrated lookups and the digital signature capability while the latter provides common web controls without the digital signature capability and lookups in a separate window. Brokers can submit and save forms, trigger workflow operations such as check in and check out, and view the forms using two types of editors.

To help achieve higher accuracy and fewer treatment errors, CFM provides the validation of

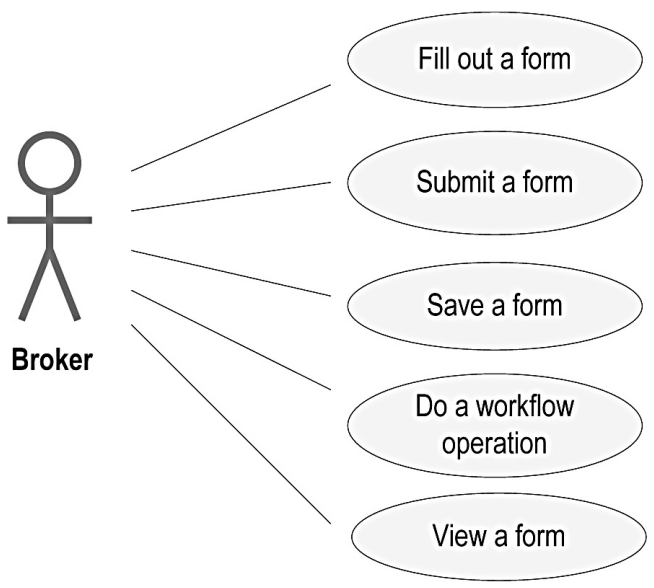

Figure 2. Basic broker use cases. entered information built into the forms. All required fields must be completed before submission, which reduces omissions. Tracking and quality control are improved because forms are time-stamped and electronically signed. Patient information captured in one department can be made available to clinical systems used in other departments, which improves the efficiency of patient discharges or transfers, order processing, and continued treatment.

\section{System Design}

In this section we elaborate core design decisions and concepts behind CFM.

The CFM collaboration functionality is based on web sites and XML editors. Editors allow an efficient collection of information from users in a structured manner. After a form has been filled out, it is submitted to a fixed storage location in one of the SharePoint site's form libraries. The submitted form represents a message in CFM - a basic unit of communication between components and servers. The BizTalk Server's mapping in concert with XML ensures the integration between healthcare participants (actors) by transforming clinical forms to the others and providing the functionalities to design, execute, and manage business processes.

\subsection{The Solution Concept}

Currently, CFM integrates the BizTalk server [3], the SharePoint Services [4], the MS SQL Server, the MS InfoPath XML editor, and ASPX pages. In addition, the framework that builds up CFM makes use of Web services, handlers and adapters providing an integrated BPM solution in a SOA manner. The communication with external systems is done using domain specific protocols such as HL7.

The Windows SharePoint Services provides intranet-based team collaboration sites that serve as a hub for vital communication and basic document management facilities. The BizTalk server enables the connection of diverse applications and communication protocols and it is suitable for both internal and external integration. BizTalk provides development and execution environments for constructing and orchestrating 


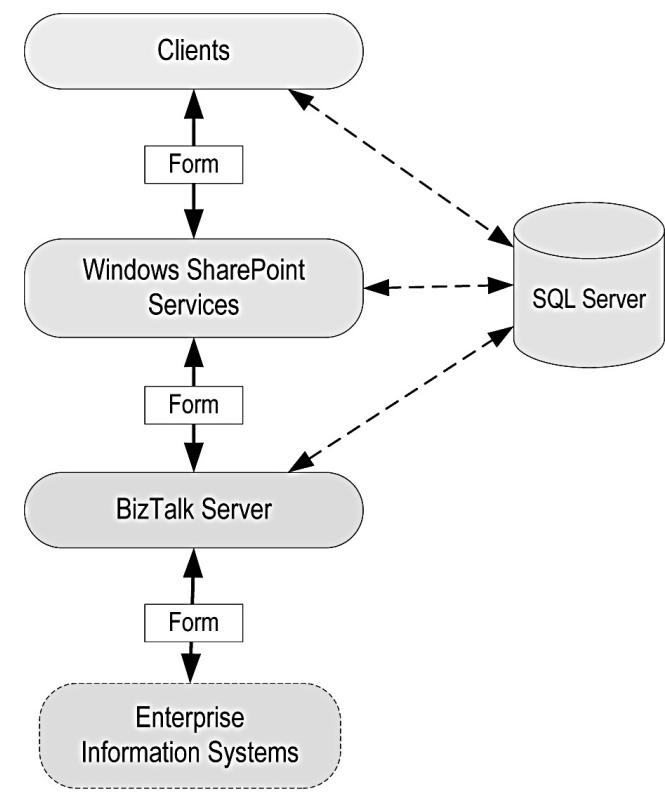

Figure 3. The CFM's solution concept.

dynamic business processes described in BEPL. The SQL Server is used for all data storage in CFM. All business data including information about patients, drugs, and etc. are stored in the database, as well as Windows SharePoint Services configuration information and CFM medical forms.

\subsection{The Logical Architecture}

The primary design choice behind CFM was how to integrate multiple servers, possibly implemented in different technologies and speaking different protocols, in a transparent yet efficient way.

With reference to Figure 4, we decided to implement two integration layers - the first CFM layer integrates editors and sites and the second one integrates sites and BEPL-based business processes. The mapping to a database is performed inside the business processes. Clinical forms (documents) are saved to a database from which custom reports can be generated. The completed documents are digitally signed and stored to the owners' sites.

The presentation layer of the CFM is built on top of the SharePoint Services web sites that are organized in a hierarchical structure. Every participant has its own site which is a centralized place for all his/her documents, information, and application accesses. To create rich

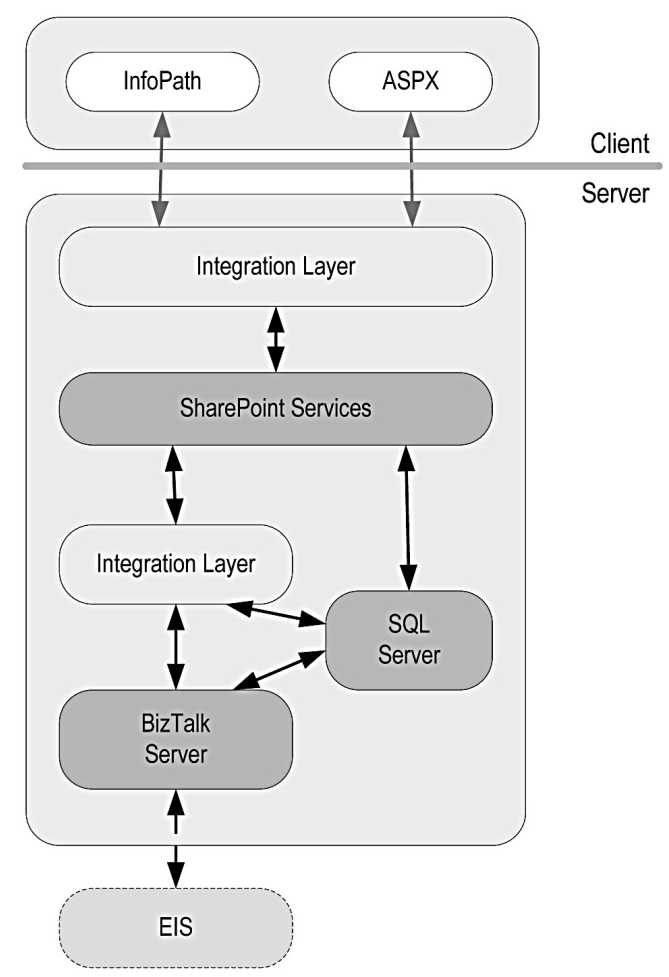

Figure 4. The CFM logical architecture.

forms, CFM clients can use the InfoPath editor or ASP.NET pages for standard forms. Completed clinical forms are submitted to the server side and are stored in appropriate SharePoint form libraries. When forms are received on the form library, the CFM automatically routes the forms, via its event handlers, through the BizTalk server, to the destination sites. Instant messaging and alerting is used to notify the recipients of CFM messages. The transformation of medical forms is accomplished by BizTalk orchestrations, which automate that process providing an easy mapping of medical forms as well as the persisting mechanisms for important data. Adapters are used to implement the communication between the orchestrations and sites.

\subsection{Security Aspects}

Application-level security in CFM utilizes Windows integrated security. The security context of SharePoint is used to access components, services and databases. If an event handler needs to be run in a different account, the user credentials are established via user impersonation. Role-based security controls access to 
detailed patient information and enables more accurate treatment and faster response times, while at the same time protecting the patient's privacy and enforcing hospital and regulatory policies.

On the CFM client, security is based on InfoPath fully trusted form security. Fully trusted forms have greater security grants and are allowed to access system resources and other components on a user's computer. Standard encryption mechanisms are employed to protect data during transmission. For example, CFM sites can be accessed using Secure Sockets Layer (SSL). In the next version of CFM we will add an Enterprise Single Sign-On (SSO) support to provide a way to map Windows user IDs to nonWindows user credentials.

\subsection{Transactions}

Based on BizTalk orchestrations, CFM groups its operations and supports both atomic transactions and long-running transactions. Atomic transactions are traditional database transactions, while long-running transactions that can span minutes, even weeks at a time, are based on compensating logic rather than on rollbacks to handle unexpected events.

\section{Implementation}

In this section we show how core CFM components: sites, orchestrations, adapters, event handlers, and web services, are implemented and used to integrate servers and editors by exchanging XML messages with one another. $\mathrm{CFM}$, as an approach for integrating distributed components, is similar to Service Component Architecture (SCA), except that CFM does not explicitly support references.

\subsection{Collaborations}

The primary design choice for CFM collaborations was to provide an easy creation, posting, and efficient exchange of clinical documents. CFM makes use of SharePoint Services to provide an easily extensible and Web-based workspace for secure communication, collaboration and document storage, thereby protecting documents and intellectual property. The basic SharePoint document management facilities are then via CFM extended by BizTalk orchestrations, forming uniform business processes.

\subsection{Forms and Documents}

Each CFM form is based on an InfoPath template and is thereby compliant to the Health Level Seven Clinical Document Architecture (HL7 CDA) [10]. A form template is a set of files that define the data structure, appearance and behavior of a CFM form. Forms are described using an XSD which specifies the hierarchical structure of the form's data and the data types that can appear in the elements. Application intelligence is intensively used to validate information or data as it is input, avoiding errors and aiding in data cleansing and normalization.

We use SharePoint form libraries as the primary integration point between SharePoint Services and CFM forms. Form libraries provide a simple way to share and track XML-based information that have a common type and, in fact, act as a form server, where users can fill out and store forms. Using form libraries, users can specify how forms are maintained, deployed, and shared for use by the authorized members. In addition, users can display columns of information extracted from the forms in order to create custom views and organize the forms and their content.

\subsection{Persistent Data}

The CFM forms, as well as other system components, store their persistent data in an SQL database. To provide reporting facilities, each CFM form is stored in a separate table and the whole document is stored as XML in a separate column. In addition, the forms are stored as documents that are digitally signed and placed in a SharePoint site where they are available for browsing to the clients.

Each lookup is implemented to access a database to provide assistance in entering valid data. For example, the forms that are sent to a pharmacy are validated to drug-drug interactions by checking a database that contains valid interactions. 


\subsection{Messages}

Enterprise servers that are integrated by CFM communicate by exchanging messages. In this context, a message is just an abstract way of looking at XML document data and, therefore, messages contain all data necessary for business functionality as well as for message routing. Every medical form is treated as one message type. It means that there are as many messages types as the form templates.

A CFM message has three main integral parts: a header, body, and signature. A message header describes information that is common to all messages such as routing. A message body specifies a unique structure for every clinical form. A message signature ensures that the message hasn't been modified by anyone else after it was completed.

\subsection{Business Processes}

In today's business environment, it is increasingly important to create business processes that combine separate and diverse applications into a coherent whole. CFM makes use of the BizTalk Server to drive business processes via its BPELbased orchestrations.

A business process is a set of actions that, taken together meet a business need. Rather than requiring expressing the various steps of CFM business processes in a programming language, we use a graphical tool to create orchestrations by connecting a series of graphical shapes in a logical way. When message is received in an orchestration - depending of a message type, an appropriate mapping component can be used to transform the source XML message to the new one. Also, in an orchestration, data from messages, as well as the whole XML documents, can be written in a database. When all actions from orchestration finish, a message is sent to a corresponding destination.

Figure 5 depicts a simple CFM business process: an orchestration receives a message of type $\mathrm{N}-001$, stores the message in a database table, creates a new message for insurance, transforms messages $\mathrm{N}-001$ to IN-001, and finally sends the newly created message to the destination. The mapping between forms data and database columns is done via the BizTalk Mapper.

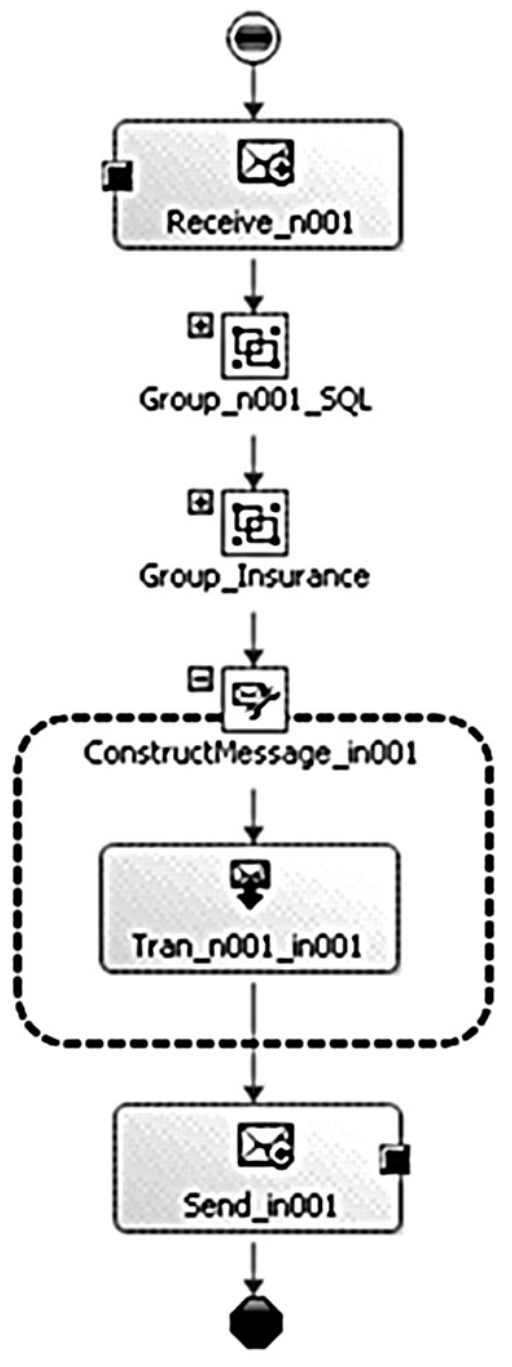

Figure 5. A CFM business process.

\subsection{Handling Events}

CFM makes use of events handlers to process events associated with document libraries. This feature is primarily used to start business processes typically by moving documents to specific locations. Currently, CFM implements two types of event handlers: the sending handler is used to send messages from document libraries to BizTalk orchestrations, while the receiving handler is used to receive messages from the BizTalk orchestrations, transform the messages, and store them into appropriate destination document libraries.

CFM event handlers are DLLs that implement IListEventSink interface. They must be placed in Global Assembly Cache (GAC) and attached 
to a document library. Handlers run within the same application pool account as the Windows SharePoint Services virtual server. The core functionality of a CFM event handler includes checking event types (e.g. CheckIn, CheckOut, ...), reading documents from corresponding sites, copying documents, impersonating users, and pushing forms to business processes.

\subsection{Connecting Existing Systems}

CFM makes use of software components called adapters to integrate the servers, as well as to connect existing systems. CFM makes use of technology adapters to integrate the BizTalk and SharePoint servers. To orchestrate data exchange with another healthcare application, CFM can leverage the BizTalk Accelerator for the HL7 standard [10] [12] and the Reference Information Model (RIM). For example, CFM is designed to operate with implementations of the HL7v3-HR specification [11] which includes definitions of core business processes that are constitutional part of the Primary Care in Croatia today.

\subsection{The SOA Glue}

There is no broad consensus around the definition of SOA. From our perspective, SOA is based on distributed software components known as services that are explicitly defined in form of interfaces and can interact with each other by exchanging messages. Service-orientation in CFM is achieved via: Web services, BEPL processes, adapters, handlers, and lookups. Web services are extensively used in CFM to send and receive XML messages (clinical forms), thereby enabling enterprise servers and components to interoperate with each other.

In certain scenarios, users might wish to fill out their forms with information that can be obtained from other parts of the system, so that users do not have to fill in repetitive data or data that is available elsewhere. That functionality is implemented through lookups, which automatically extend clinical forms' information via web services that obtain data from databases. For example, drug-drug interactions are implemented using implicit lookups.
CFM provides online approaches to monitor business processes it delivers, by visualizing how they are performing via Business Activity Monitoring (BAM) facilities.

\section{Experimental Environment}

Our experimental test bed consists of several interconnected computers (nodes) - a CFM node can be a CFM server running CFM server components or a CFM client running a browser or an InfoPath editor. On each CFM server node the following software is installed: the Windows Server 2003, the SharePoint Services 2.0, the BizTalk Server 2006, the SQL Server 2005, and the .NET Framework 2.0. CFM clients run different Windows-based operating systems, a browser and optionally an InfoPath editor.

The topology of our interconnected network containing CFM nodes is similar to National Health Information Network (NHIN) [14] since each region is represented by a CFM server which supports several healthcare purchases and providers (hospitals, labs, clinicians) and payers. Servers can communicate with each other to exchange information via common standards and data formats. Both CFM clients and servers can be added and removed from the network, allowing CFM to grow dynamically.

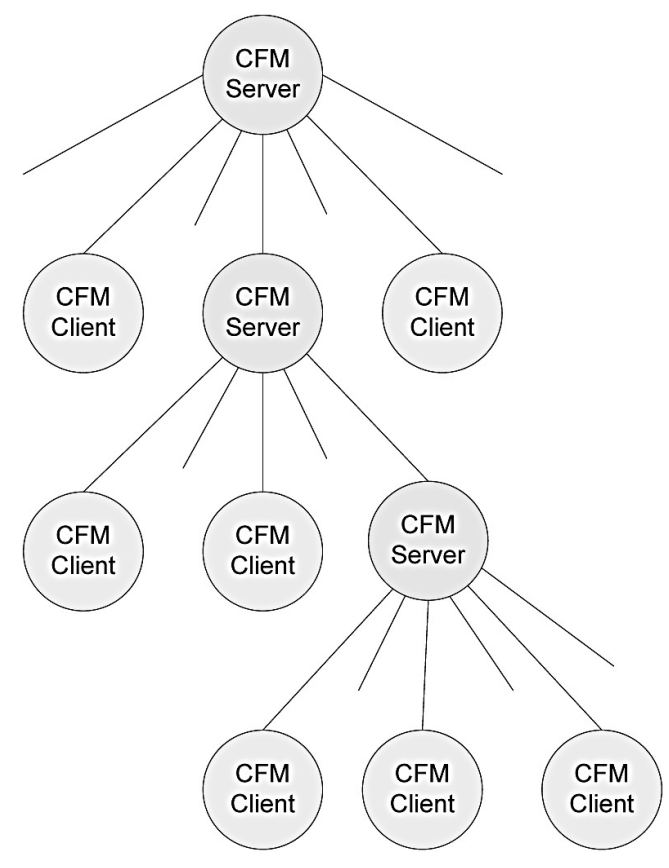

Figure 6. The network topology of CFM. 


\section{Related Approaches}

In this paper we present important technological aspects and tradeoffs that we applied in the development of medical business processes supporting medical forms and documents. To compare our solution to other approaches in medical informatics, we use the following criteria: (1) the software architecture, (2) the tool support, (3) the underlying platform, (4) support for IT standards in medicine, and (5) user experience. Tele-health aspects are out of the scope of our project and this paper.

The software architecture applied in medical informatics encompasses software layers and servers. Most approaches support three layers: the presentation layer, the business layer, and the data layer. Software servers greatly reduce time required to develop BMP solutions. CFM utilizes server technologies to support and automate development in all the layers. SharePoint Services and IIS (Internet Information Services) support objects used in the presentation layer, the BizTalk and SQL servers support business and data layers allowing data mapping and transformation. Some approaches such as [11] do not utilize software servers and develop all software components from scratch, while the others such as [13] utilize software servers and frameworks.

Tools can be applied in early phases of the Software Development Lifecycle (SDLC) such as design and mapping tools and in later phases of SLDC such as monitoring and performance analysis tools. CFF makes use of software tools in all the phases of the SDLC including modeling business processes, mapping business processes to persistent store, and monitoring the health of business processes.

The underlying platforms used in medical IT solutions are native platforms such as Windows and UNIX, and virtual machine platforms such as .NET and Java. Most modern software solutions are based on virtual machines. CFM is based on .NET, since at the beginning of our development we choose the BizTalk server as a BPM server and it is based on .NET.

Most IT solutions in medicine support standards such as HL7, RIM, EHR (Electronic Health Record), and SOA standards such as WSDL, SOAP, and WS-*. CFM supports core SOA standards and has adapters for HL7 and EHR. Internally, CFM works on forms that are stored as digitally signed documents, but makes use of database triggers to transfer data to our EHR database.

Modern IT medical solutions are Web-based and utilize common Web technologies such as HTTP, HTML and AJAX. Some solutions make use of rich editors such as the InfoPath editor. CFM supports both approaches and provides Web-based forms that provide UI functionality without digital signature, as well as rich forms which include digital signature. Both types of forms incorporate security mechanisms required in a distributed environment.

\section{Conclusion}

Both the design and implementation of the CFM solution providing a framework for managing clinical forms and corresponding business processes have proven to be very successful and beneficial. CFM demonstrates how to use highlevel software technologies to automate the process of collecting, organizing, sharing, and managing clinical forms and other information in healthcare organizations.

CFM integrates server technologies to implement business processes and therefore reduces time needed to build, deploy, and manage such class of applications. Additional flexibility is achieved by the possibility to easily add new clinical forms or change the existing ones.

CFM forms are XML-based documents that can be created by two different editors. Documents' repository is implemented via SharePoint Services. The core logic that drives business processes is implemented using BizTalk orchestrations that obtain clinical forms, perform the requested data transformations, store data into the database, and push forms further into business processes. Integration between servers and software components is realized via Web Services, adapters, and event handlers. SharePoint event handlers receive forms from orchestrations and, according to the routing information, deliver them to recipients. Web services provide inputs and outputs for orchestrations and implement forms' lookups.

Our solution is based on core IT and medical standards and can therefore be connected 
to other medical information networks, thereby fostering collaboration in medical information systems.

This paper has discussed issues and solutions for the implementation of healthcare services utilizing advanced software technologies. Most of our efforts in that regard have been to implement documents and business processes in a portable manner utilizing server technologies and standards such that CFM can be easily ported on other platforms.

\section{Acknowledgment}

We would like to thank the Business Innovation Center of Croatia (BICRO) for fully founding the project whose technological aspects are described in this paper.

\section{References}

[1] W. M. P. VAn der Aalst, A. H. M. DER HofstEDE, M. WESKE, Business Process Management: A survey. In Proceedings of the 2003 International Conference on Business Process Management (BPM2003). Lecture Notes of Computer Science 2678. Berlin, Springer-Verlag, pp. 1-12, 2003.

[2] W. M. P. van DER Aalst, A. H. M. TER Hofstede, B. KIEPUSZEWSKI, A. P. BARROS, Advanced Workflow Patterns. In O. Etzion and P. Scheuermann, editors, 7th International Conference on Cooperative Information Systems (CoopIS 2000), Vol. 1901 of Lecture Notes in Computer Science, pp. 18-29. Springer-Verlag, Berlin, 2000.

[3] Scott Woodgate, Stephen Mohr, Brian LoesGEN, Microsoft BizTalk Server 2004 Unleashed. Sams Publishing, 2005.

[4] SCOT P. HILliER, 'Microsoft SharePoint - Building Office 2003 Solutions'. Apress, 2004.

[5] ThOMAS ERL, Service-oriented Architecture: Concepts, Technology, and Design. Prentice Hall, 2006, ISBN-10: 0131858580.

[6] DAVID ChapPELL, Understanding BizTalk Server 2006, White Paper, 2005.

[7] BoJAn Blazona, ŽElJKa MiHAJlović, Visualization Services Based on Web Services, Journal of Computing and Information Technology, Vol. 15, No. 4, 2007, 339-345.

[8] SRINIVAS PADMANABhUni, JAi GANESh, DEEPENDRA MOITRA, Web Services, Grid Computing, and Business Process Management: Exploiting Complementarities for Business Agility, IEEE International Conference on Web Services (ICWS'04), pp. 666, 2004.
[9] IVAn Zoraja, Ivan Zulim, Maja Štula, CORAL - Online Monitoring in Distributed Applications: Issues and Solutions. Wseas transactions on computers. Issue 3, Vol. 7, March 2008.

[10] Online, http://www.hl7.org/

[11] HL7v3-HR Specifications, No. 12/15519-FCPBA 10124 Uen, Ericsson 2004.

[12] Goerge W. Beller ET AL, Message Development Framework, v3.3. by Health Level Seven, 1999.

[13] Clinical Document Architecture (CDA) for HL7, Microsoft Corporation.

[14] ANNA O. ORLOVA, National Health Information System. Presentation, John Hopkins School of Medicine, March 2007.

Received: March, 2008 Revised: September, 2008 Accepted: October, 2008

Contact addresses: Ivan Zoraja Boris Ilijic FESB

Dept. of Electronics \& Computer Science University of Split R. Boskovica bb, 21000 Split Croatia e-mail: zoraja@fesb.hr

Vojimir Golem MicroGolem Trg Hrvatske Bratske Zajednice 8/II 21000 Split Croatia

IVAN ZORAJA is a professor at the University of Split, Croatia and a director of Zoraja Consulting company. He received his Ph. D. degree in computer science from the Technical University of Munich, Germany. His primary research interests are software engineering, distributed systems, and business processes.

VOJIMIR GOLEM is a software architect and a director of MicroGolem, Croatia. He received his diploma in computer science from FESB, University of Split, Croatia. His primary research interests are project management, business processes, and document management.

BORIS ILIJIĆ is a research assistant at the University of Split, Croatia. He received his diploma in computer science from FESB, University of Split, Croatia. His primary research interests are mobile systems, business processes, and web portals. 
